\title{
Immediate correction and adaptation based on viewing a prismatically displaced scene'
}

\author{
IRVIN ROCK, JOEL GOLDBERG AND ARIEN MACK \\ YESHIVA UNIVERSITY
}

It was shown that when observers view a scene of a room through displacing prisms there is an immediate correction of the prismatic distortion. Objects appear to lie in a direction closer to the ir true direction than to that produced by the refraction of the prisms. It was also shown that a brief period of exposure to the prismatically viewed scene, without movement or sight of the body, results in substantial adaptation to the displacement.

If an observer looks through a wedge prism which displaces light sideways, objects in the scene do not appear as displaced as they ought to, given the magnitude of the displacing power of the prism. This phenomenon can easily be observed by first noting the apparent direction of an object seen through the prism with one eye and by then opening the eye which is not looking through the prism. The exact magnitude of optical displacement can be detected by noting where in the non-prism scene the object viewed through the prism is located. It will be noted that the object seen through the prism had appeared far less displaced than it actually was displaced by the prism. For those who find it difficult to perform this binocular test, an alternative is first to look through the prism, note where the object appears, and then quickly remove the prism and note where the eyes are actually pointing. This effect is perhaps even stronger when the prism displaces in an upward or downward direction. An object at eye level seen through prisms appears to be almost at eye level despite the fact that the eyes must be very considerably raised or lowered to see it through the prisms. In the light of all the research done on prism displacement in recent years, it is surprising this phenomenon has not previously been noted.

This immediate correction of prismatic displacement is a function of seeing the entire array of objects in the room. If only a single luminous target is viewed through prisms, it will appear displaced by precisely the amount that the prisms deflect the rays of light. In our experiments, therefore, we took as a measure of the correction effect the difference in apparent direction of a target on the wall seen with lights on and with lights off.

The explanation of this correction effect is not at all obvious. Of course, if an observer knows his position and orientation in the room prior to looking through the prisms, then one might conclude that this is the basis for objects continuing to appear to be located where, in fact, the observer knows them to be (although, if true, such an effect of knowledge on perception would still be surprising). But in our work the observer is always led to his position blindfolded, thus ruling out such an explanation. Despite this, the observer does obtain certain kinds of information as to approximately where in the room he is. For example, a wall on his left, parallel to the sagittal plane of his body, may, when viewed through the prisms, be optically shifted to a point where it ought to appear straight ahead. But, if it were straight ahead, it would no longer project the image that it now does project-the trapezoidal image of the wall would compress to a line-and hence the observer is receiving information that the wall is off to his side. However, the observer might then experience his own orientation to the wall as different than it actually is. He might experience himself as turned in space about a vertical axis in one direction by precisely the amount the prisms displace the field in the other direction, since this misperception would account for the visual scene. If he did thus misperceive his own orientation, there would be no correction effect. Whatever the explanation, we believe the effect is based on seeing a scene such as a room in three dimensions. If only the opposite wall were visible-that is if the floor, ceiling, and other walls were not visible-it is difficult to see how this view would differ from that of a single point. In fact we have found that when all that can be seen is an array of small luminous shapes arranged randomly over the surface of the entire front wall, there is no correction effect.

The effect is, however, more easily explained when the prismatic displacement is upward or downward. Parallel to what was said above concerning lateral displacement, one might say that the impression of an upward-displaced room could be accounted for by the observer if he were to experience himself-or just his head-as tilted downward. But this will not happen if, via proprioceptive information based on gravity, the observer were to experience his own posture veridically. If, further, there is a tendency for the floor and ceiling of the room to be seen as spatially horizontal-because the main axes of the room serve as a determinant of the vertical and horizontal-then the correction effect becomes understandable. Since the observer feels he is perpendicular to the floor, and therefore parallel to the walls, only a point on the wall which is at eye level would appear to be at eye level. Of course in the above discussion we have disregarded the role of eye position in determining the radial direction of a retinal point. Ordinarily, an object will appear to be at eye level when 
the eyes are horizontal. Therefore the prism situation described is one of a conflict between the information derived from the structure of the scene leading to the correction effect and information concerning eye position.

\section{THE CORRECTION EFFECT}

\section{Method}

The procedure of the experiments we have performed on this effect is as follows: The observer is brought blindfolded into the laboratory room. $\mathrm{He}$ is required to bite down on a teeth mold so that he remains in fixed position throughout. With the room totally dark he then positions a small luminous spot focused on the wall facing him by moving a handle which turns a slide projector until the spot appears straight ahead (in some experiments) or at eye level (in other experiments). The average setting made by the observer serves as an index of the position of the spot which, for him, appears straight ahead or at eye level. Next the prism spectacles are placed on the observer and again he adjusts the position of the spot. This provides a measure of the displacement produced by the prisms. Since we used prisms of 20 diopters, it is to be expected that the spot would, on the average, be set about $11^{\circ}$ from the position selected without prisms. Next, the room lights are turned on and the observer again positions the spot. The difference between this and the preceding condition in setting of the spot is a measure of the correction effect. The observer can not see any part of his body since the board to which the teeth mold is attachedblocks it from view. Following this, the prisms are removed, the lights are turned out, and the observer positions the spot once more. This step is not immediately relevant to the aspect of the experiment under discussion. In each step outlined above, four settings are made by the observer, alternating the starting position of the spot on the left or right (above or below). The position of the spot is indicated to the experimenter by a protractor mounted below an arm which moves with the projector.

\section{Results}

The results of one experiment in which the prismatic displacement was upward or downward was as follows. When the prisms displaced objects in an upward direction, the average setting of the spot in the dark for 12 observers was $12,4^{\circ}$ below the average position selected by the observers without prisms (which was quite close to the objective eye level position). When, however, the lights were on, the average setting was only $3.9^{\circ}$ below the (subjective) eye level position. This means that on the average observers are selecting a position of the spot as appearing at eye level which requires the eyes to be turned upward by about $7.1^{\circ}$ (since the prisms displace all points upward by $11^{\circ}$ ). The average difference between settings in the dark and with lights on was $8.5^{\circ}$. For downward displacement of the prisms, for a separate group of 12 observers, the average setting of the spot in the dark was $10.5^{\circ}$ above the (subjective) eye level position. With lights on, the average setting was $4.7^{\circ}$ above that position. Hence on the average the observers are here selecting a position of the spot as appearing at eye level which requires the eyes to be turned downward by about $6.3^{\circ}$. The average difference between settings in the dark and with lights on was $5.8^{\circ}$. Every one of the 24 observers in the two experiments combined showed the correction effect.

Comparable results were obtained for sideways displacement of the prisms. The observer displaced the spot until it appeared straight ahead of his nose by moving the spot along a horizontal path. The observer was seated in these experiments (he was standing in the experiment on vertical displacement). The chair was placed so that the sagittal plane of the observer's body was perpendicular to the opposite wall. Many variations of this experiment were performed and a large correction effect was always obtained for both left and right displacement of the field. The average difference between placement of the spot in the dark and with lights on was between 6 and $7^{\circ}$ when the observer was in the center of the room and somewhat smaller when he was off to the side of the room.

It is possible to argue that the correction effect is based on a tendency for the direction which appears to be straight ahead to be one which is also centered in the visual field (see Dietzl, 1924; Roelofs, 1935)。This would operate when the observer in our experiments is in the center of the room. However, we have shown that this is not a necessary factor by placing the observer on the left side of the room with prisms oriented to displace objects leftward. We still obtained a marked correction effect despite the fact that here it occurs in the direction opposite to any such centralizing tendency. However, the effect was smaller than when the observer was in the center of the room. Harris et al (1966) have suggested that there may be a tendency for the direction which appears straight ahead to be one which is perpendicular to the wall facing the observer. If, therefore, the direction which appears to be perpendicular to the wall through the prisms is the one which is objectively straight ahead, the correction effect could in actuality be the result of such a bias rather than of information as such. However, in assessing the relevance of such an explanation, we tested observers without prisms, placing them at various orientations with respect to the wall, and found no evidence for a "perpendicular bias." Further, we obtained the correction effect (with prisms) when the orientation of the observer with respect to the wall was systematically varied.

\section{ADAPTATION}

We turn now to a further question. If-for whatever reason-there is immediate correction of the prismatic displacement, then the conditions exist for the kind of adaptation which can be expected to yield an aftereffect. The egocentric localization or radial direction of objects 
is a joint function of retinal locus and position of the eyes. Ordinarily, an object whose image falls on the fovea will appear to be at eye level only when the eyes are horizontal. If now, in looking through prisms, the object appears at eye level when the eyes are turned upward, then an association may develop between that position of the eyes and the phenomenal location "eye level," for objects stimulating the fovea.

\section{Method}

To test this, it was only necessary to extend the period of exposure to prisms with room lights on beyond the point described above where the four settings were made. This period lasted anywhere from $1 \mathrm{~min}$. to 10 min. depending upon the experiment. The observer remained stationary and merely viewed the scene. The pre- and post-exposure settings of the spot made in the dark with prisms off-referred to above-constituted the measure of adaptation.

\section{Results}

The results are best expressed in terms of the difference in degrees in the average setting of the spot before and after exposure to the prismatically viewed scene of the room. The prediction is that the shift will be in the same direction in which the prisms displaced the field. In the experiment on vertical displacement for which the results of immediate correction were given above, the average shift for the 12 observers for upward displacement after a $5 \mathrm{~min}$. exposure period was $3.0^{\circ}$ in the predicted direction; for downward displacement, it was $2.0^{\circ}$. Combining the two groups, the average shift was $2.5^{\circ}$ which yields a $t$ significantly different from $0(p<.01)$. When only the first two of the post-exposure settings are averaged the effect is generally even greater, so that there would seem to be a rapid wearing off of the adaptation achieved. The majority of observers show a change in the predicted direction, and for some the change is very appreciable. There are, however, a few who either show no change at all or a slight change in the opposite direction. This adaptation effect is reliable since we have obtained it repeatedly in variations of the experiment, most of which entailed left or right prismatic displacement. In one such experiment, following a $10 \mathrm{~min}$. exposure period with the observer in the center of the room, the average shift for 17 observers was slightly under $4^{\circ}$; it was over $4^{\circ}$ when only the first two post-exposure settings were compared with the pre-exposure settings.

It might be argued that the adaptation effect obtained is based on a process of normalization of an atypical posture of the eyes rather than on information concerning phenomenal location. If most of the time the observer tends to look at the region which is central in the prism scene, then most of the time his eyes will be turned to the side. Assuming this to be true and that, further, this position begins to "feel" as if it were straight ahead simply because the eyes have been in that position for some time, one would have to predict an aftereffect such as we have obtained. That there is some basis for this argument is borne out by an experiment we performed in which the observer, without prisms, was required to fixate $11^{\circ}$ to the side for a period of $5 \mathrm{~min}$. An aftereffect was obtained when only the first two post-exposure settings were considered. Therefore, to demonstrate that atypical eye position was not the necessary basis of the effects obtained in all other experiments, a control experiment was performed. During the phase of exposure to the room with lights on, the observer, wearing prisms, was required to fixate that point in the field which necessitated that his eyes be physically straight ahead. Since the prisms displaced objects by $11^{\circ}$, the point fixated was $11^{\circ}$ to the side of the head opposite to the direction of prism displacement. Otherwise the procedure was the same as in the other experiments described. The same observers also served in the condition where they were not required to fixate. There was no apparent difference in the results of the two conditions. Adaptation occurred in both cases.

\section{DISCUSSION}

It is worth emphasizing that a shift of 2 to $4^{\circ}$ in egocentric localization such as we have obtained is greater than that typically found in studies of adaptation to displacement even where the period of exposure is much longer than ours (see Held \& Bossom, 1961; Weinstein, Sersen, Fisher, \& Weisinger, 1964). This is all the more interesting when it is realized that our observers do not see any part of their own bodies and do not move, factors which have been thought to be essential to adaptation. If, however, as we believe, visual adaptation to an optically displaced image consists of forming new associations between specific positions of the eye and information concerning phenominal location of objects vis-a-vis the self, then our procedure is indeed ideal for establishing adaptation (see Rock, in press). For whatever reason, information exists for our observers from the outset that objects are not where the optical displacement dictates they ought to appear to be.

Whereas in the case of information derived from other sources, as for example from movement, it is not the case that movement immediately makes evident the nature of the prismatic distortion. The information is undoubtedly supplied, but it must be extracted over time. However, it should be noted that in previously reported research, whenever the observer can see the room or corridor, the correction effect should be present and should lead to adaptation. It is possible that such an effect accounts for the very appreciable adaptation obtained in an experiment by Wallach, Kravitz, and Lindauer (1963) in which the observer in a supine position merely viewed his legs for a period of $10 \mathrm{~min}$. Presumably the observer could also see beyond his legs across the room, so that the conditions for a correction effect 
may have been present. But by the same token, it is somewhat of a mystery why the effect we obtained in 5 or $10 \mathrm{~min}$. is greater than that obtained in an hour by Held and Bossom or Weinstein et al, unless movement confounds the correction effect rather than augmenting it.

Given the immediate correction, as far as adaptation is concerned, it is irrelevant what the cause of the correction is. We would predict that "adaptation" would occur if the observer inspected certain patterns directly -that is, not through prisms-for example, a large rectangle placed eccentrically so that one of its sides was straight ahead. The direction seen as straight ahead would, as a consequence of the Roelofs effect, shift toward the center of the rectangle. That being the case, a turned position of the eyes would be associated with the impression that the fixated region-the center of the rectangle-was straight ahead. However, such an effect would be the result of a bias and, therefore, would not be veridical, whereas adaptation to displacing prisms is presumably based on information and, with prisms on, is veridical.

\section{References}

Dietzl, H. Untersuchungen uber die optische Lokalisation der Mediane. Z. f. Biol., 1924, 80, 289-316.

Harris, C. S., Harris, J. R., \& Karsch, C. W. Shifts in pointing "straight ahead" after adaptation to sideways-displacing prisms, paper read at Eastern Psychological Association, New York. April, 1966.

Held, R., \& Bossom, J. Neonatal deprivation and adult rearrangement: complementary techniques for analyzing plastic sensorymotor coordinations. J. comp. physiol. Psychol. 1961, 54, 33-37.

Rock, 1. The nature of perceptual adaptation. New York: Basic Books, in press.

Roelofs, C. O. Optische Lokalisation.. Arch. Augenheilk, 1935, $109,395-415$.

Wallach, H., Kravitz, J. H., \& Lindauer, J. A passive condition for rapid adaptation to displaced visual direction. Amer.J.Psychol., 1963, 76, 568-578.

Weinstein, S., Sersen, E. A., Fisher, L., \& Weisinger, M. Is reafference necessary for visual adaptation? Percept. mot. Skills, $1964,18,641-648$.

\section{Notes}

1. This work was supported by Grant GB 3410. National Science Foundation.

(Accepted for publication August 18, 1966.) 